\title{
Teledyne e2v sensors for adaptive optics wavefront sensing on ELTs: high rate, large format, and low noise
}

\author{
Paul Jorden*, Denis Bourke, Ryan Cassidy, Martin Fryer, Paul Jerram, Stuart Moore, Jérôme \\ Pratlong
}

Teledyne e2v (UK), 106 Waterhouse Lane, Chelmsford, Essex, CM1 2QU, UK

\begin{abstract}
Teledyne $\mathrm{e} 2 \mathrm{v}$ has a well-established heritage of designing and supplying high performance sensors for professional astronomy. These CCD and CMOS image sensors are back-thinned for maximum quantum efficiency, designed for lowest read-noise, and optimised for high frame rate use.

CCDs are appropriate cost-effective solutions for modest formats (e.g. 240 X 240 pixels) but CMOS architectures are required for larger formats at high frame rates.

We describe key elements of a major proposed sensor design for the E-ELT; this "LVSM" detector has 800 X 800 pixels in a design that is optimised for ELT use.

High rate CMOS sensors use multiple parallel ADC blocks and deliver very low pixel noise coupled with very low noise ADC circuits. These sensors provide high sensitivity at low signal levels for natural and laser guide star applications.

We present examples of sensor designs ranging from 80X80 to 1600X1600 pixels in simple and more complex Peltiercooled packages. Sensor performance considerations such as intra-pixel response, cross-talk, uniformity, data paths, and others are discussed.
\end{abstract}

Keywords: adaptive optics, wavefront sensor, CCD imager, CMOS imager, high frame rate, large format, low noise

\section{INTRODUCTION}

In this paper we discuss silicon detectors as wavefront sensors for adaptive optics (AO) systems- primarily for Shack Hartmann sensors. Charge Coupled Detectors (CCDs) are well established for this purpose on many telescopes worldwide. As telescopes increase in size the sensor format increases and the CMOS (Complementary Metal Oxide Semiconductor) architecture becomes necessary. CMOS sensors offer electro-optical performance which is comparable to that of CCDs and exceeds the CCD in data rate.

A new large-format CMOS detector for this application is presented together with examples of other specialized CMOS sensors for astronomy and space applications. Most CCD and CMOS sensors presented here are back-illuminated and anti-reflection coated for maximum quantum efficiency. Teledyne e $2 \mathrm{v}$ has also developed significant capacity for supply of sub-systems for space and astronomy applications- a few examples are illustrated.

\section{CCD AND CMOS SENSORS FOR ADAPTIVE OPTICS}

\subsection{Sensor evolution: CCDs -> CMOS}

Since the first uses of AO on telescopes Teledyne-e2v has supplied high performance CCDs. These have low noise, high spectral response, and high frame rate. Frame rates are typically in the $200-1000$ frames per second range. CCDs are well established for this purpose on many telescopes and modest sized traditional CCDs can read out at adequate rates with low noise. 
Large telescopes have more sub-apertures (SA) and need larger sensors; such sensors have more pixels and these prove difficult to read out at adequate frame rates. Increasing the pixel frequency increases the read-noise to an unacceptable level; however this can be overcome by the use of EM (electron-multiplying) CCDs which have sub-electron read-noise even at high pixel rates.

As we progress to ELT-class telescopes the readout of large areas at high frame rates become impractical for CCDs- the data rate and number of outputs is too high and power consumption is too large. The use of CMOS sensors offer large format, high frame rate, and low read noise.

The figures below illustrate a progression of designs over approximately two decades (including several company name changes).
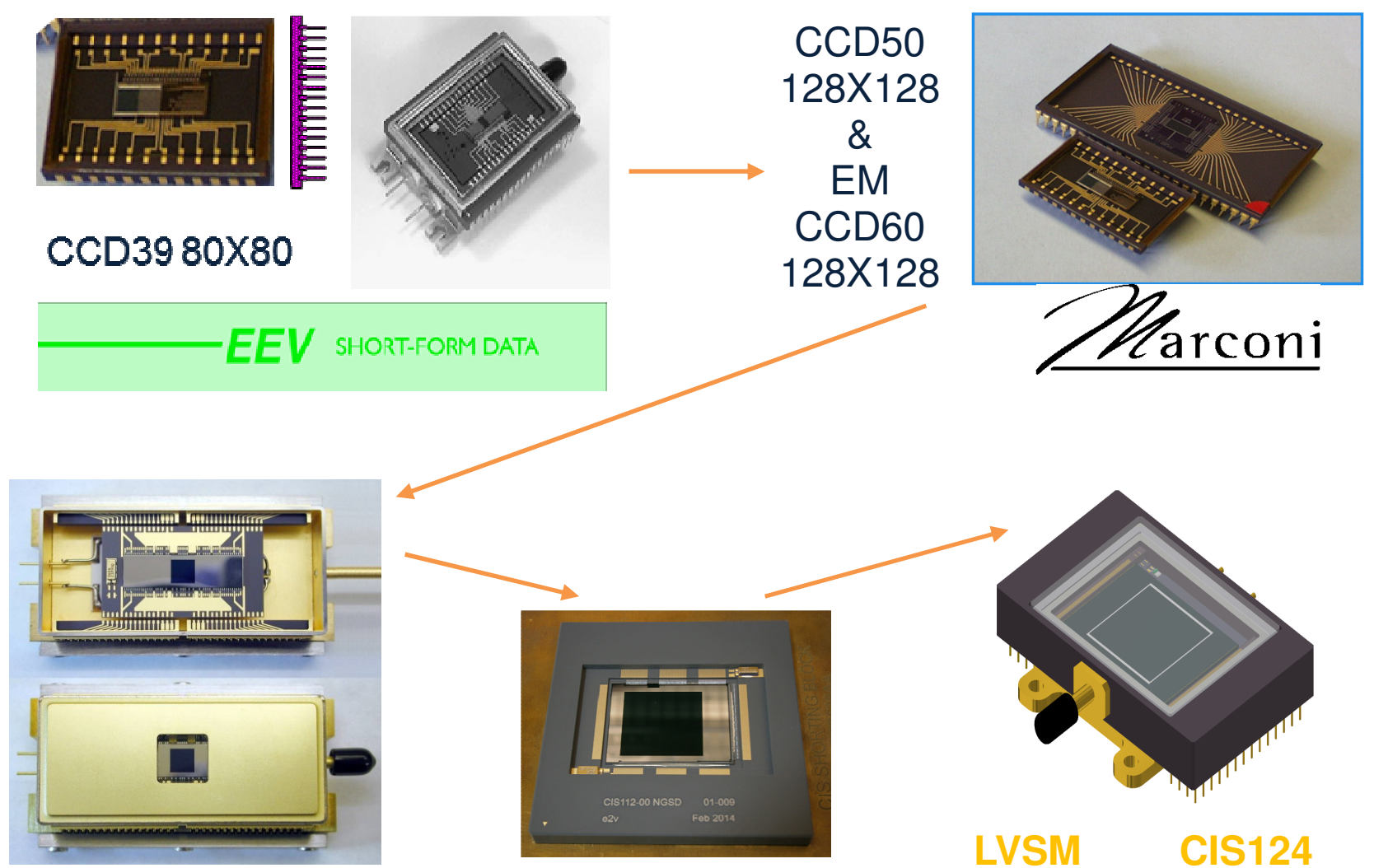

EM CCD220

NGSD CIS112

LVSM
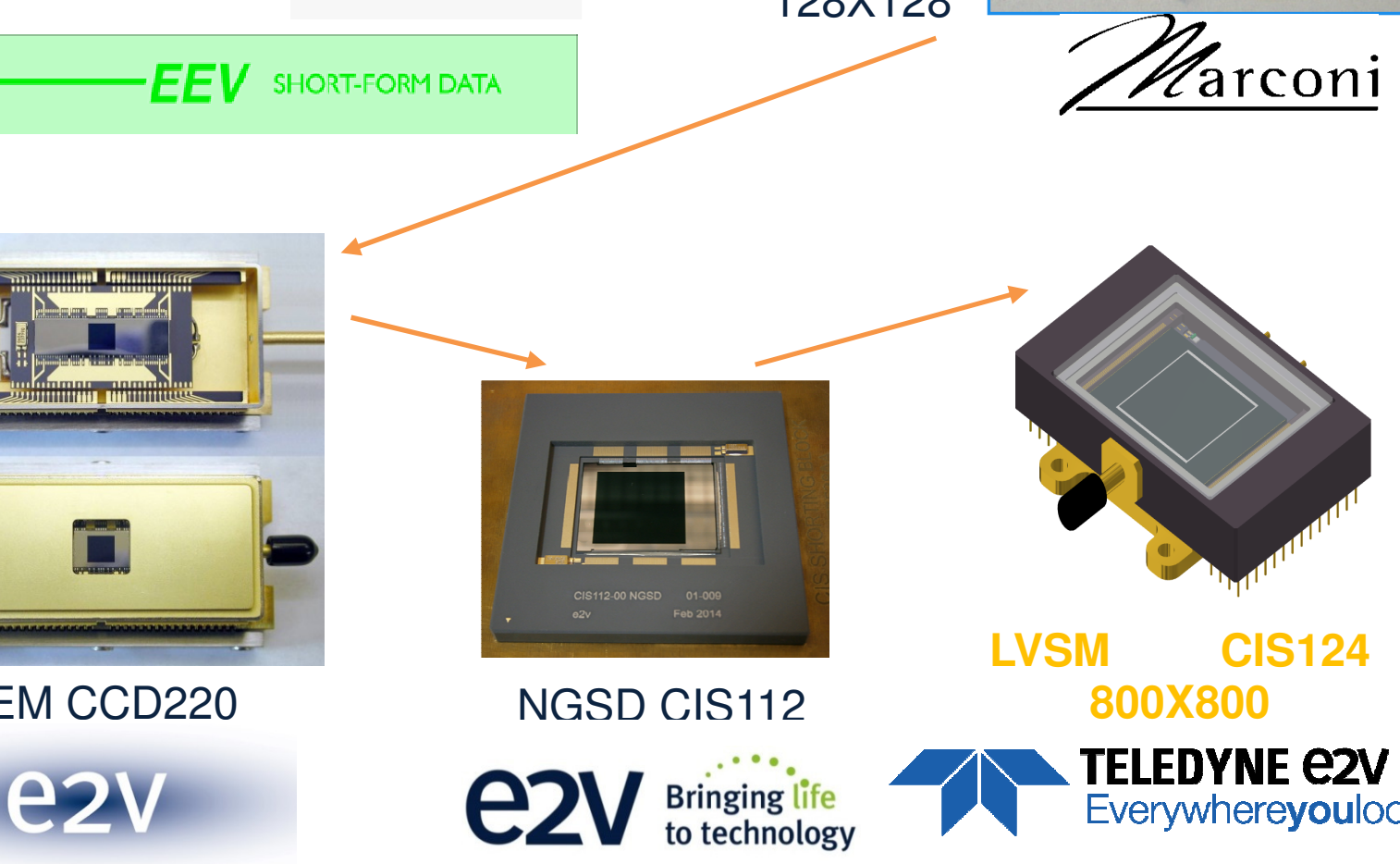

\section{$800 \times 800$}

CIS124

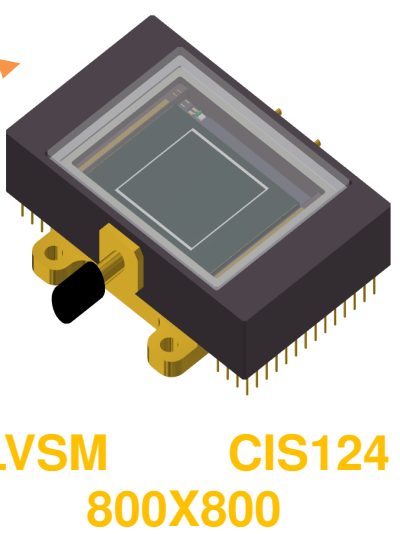

TELEDYNE e2V

Everywhereyoulook'

e2v technologies

Figure 1. Progression of WFS detector sizes

The following section tabulates key parameters of these detectors.

\subsection{CCD and CMOS wavefront sensor summary}

The table below summarises key parameters of sensors for AO. All sensors have 24 X $24 \mu \mathrm{m}$ pixels and $90 \%$ backthinned QE. See web site for datasheets ${ }^{1}$. 
Table 1. CCD and CMOS WFS detectors

\begin{tabular}{|l|l|l|l|l|l|l|l|}
\hline $\begin{array}{l}\text { Part } \\
\text { Number }\end{array}$ & $\begin{array}{l}\text { Image } \\
\text { Format }\end{array}$ & Type & $\begin{array}{l}\text { Read-noise } \\
\text { e rms }\end{array}$ & $\begin{array}{l}\text { Frame rate } \\
\text { Frames/sec }\end{array}$ & Outputs & Package & Status \\
\hline CCD39-01 & $80 \times 80$ & $\begin{array}{l}\text { Standard } \\
\text { CCD }\end{array}$ & $\begin{array}{l}5 \text { @ } 1 \\
\text { MHz/pixel }\end{array}$ & 500 & 4 & $\begin{array}{l}\text { Ceramic or } \\
\text { Peltier }\end{array}$ & Standard \\
\hline CCD50 & $128 \times 128$ & $\begin{array}{l}\text { Standard } \\
\text { CCD }\end{array}$ & $\begin{array}{l}5 \text { MHz/pixel } \\
\text { M } 1\end{array}$ & 1000 & 16 & Ceramic & $\begin{array}{l}\text { Custom } \\
\text { [superseded] }\end{array}$ \\
\hline CCD60 & $128 \times 128$ & EM CCD & $<1$ & $\sim 1000$ & 1 & Ceramic & Standard \\
\hline CCD220 & $240 \times 240$ & EM CCD & $<1$ & $>1000$ & 8 & Peltier & Standard \\
\hline $\begin{array}{l}\text { CIS112 } \\
{[\text { NGSD] }}\end{array}$ & $880 \times 840$ & CMOS & $<4$ & 700 & $\begin{array}{l}20 \\
\text { LVDS }\end{array}$ & Ceramic PGA & $\begin{array}{l}\text { Previous } \\
\text { development }\end{array}$ \\
\hline $\begin{array}{l}\text { CIS124 } \\
{[\text { LVSM] }}\end{array}$ & $800 \times 800$ & CMOS & $<3$ & 700 & $\begin{array}{l}20 \\
\text { LVDS }\end{array}$ & Peltier & $\begin{array}{l}\text { In } \\
\text { development }\end{array}$ \\
\hline
\end{tabular}

\subsection{A new electron-multiplying sensor- The CCD351}

In addition to the CCDs shown above, Teledyne-e2v has recently introduced a new standard sensor to its range. The sensor offers sub-electron readout noise and high spectral response at 30 frames/second. Further information is shown in the figure and table below.
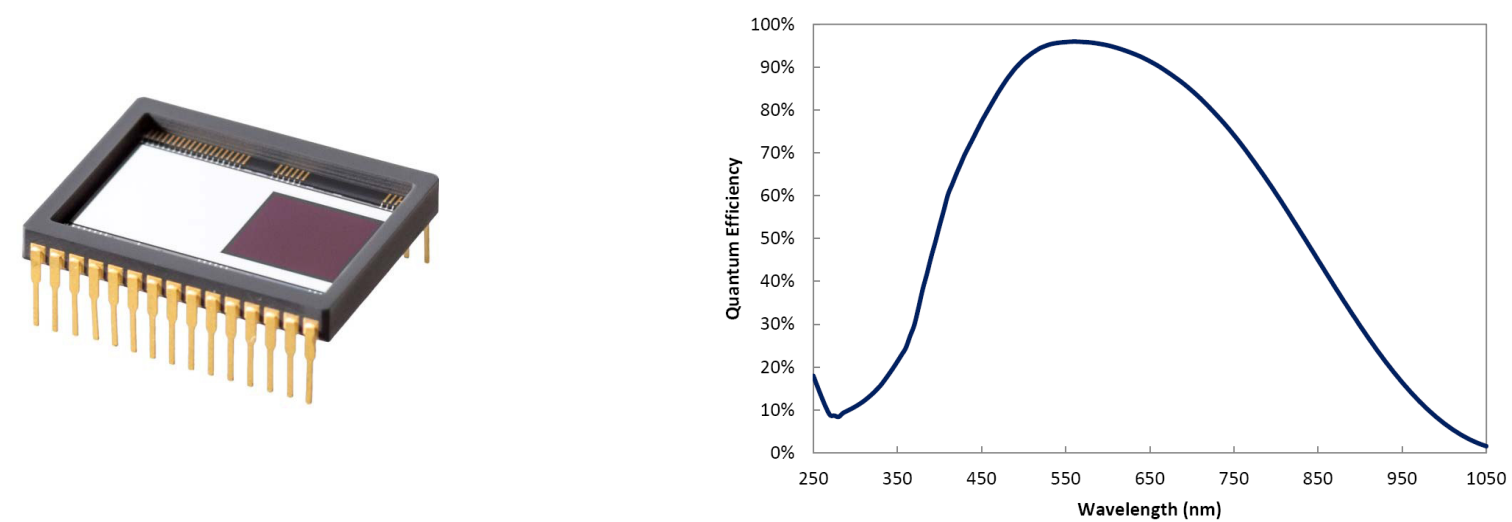

Figure 2. Sensor photo and spectral response.

Table 2. Typical performance

\begin{tabular}{|l|l|}
\hline Image section: & $1024 \times 1024$ \\
\hline Pixel size: & $10 \mu \mathrm{m} \times 10 \mu \mathrm{m}$ \\
\hline Active image area: & $10.24 \times 10.24 \mathrm{~mm}$ \\
\hline Ceramic DIL Package size: & $22.86 \times 28.00 \mathrm{~mm}$ \\
\hline Amplifier responsivity: & $3.5 \mu \mathrm{V} / \mathrm{e}^{-}$ \\
\hline Readout noise: & $<<1 \mathrm{e}^{-}$with EM gain. $50 \mathrm{e}^{-}$at unity gain \\
\hline Multiplication gain: & $100-1000$ typical (variable) \\
\hline Output data rate: & $37 \mathrm{MHz}$ \\
\hline Pixel charge storage: & $35 \mathrm{ke} / \mathrm{pixel}$ \\
\hline Dark signal $\left(18^{\circ} \mathrm{C}\right):$ & $100 \mathrm{e}^{-} / \mathrm{pixel} / \mathrm{s}$ \\
\hline
\end{tabular}




\section{THE LVSM SENSOR FOR THE ELT}

\subsection{Introduction}

Teledyne-e $2 \mathrm{v}$ is currently designing a new high performance sensor for use on the European ELT and other similar-sized telescopes. Key design features are itemized below-

- $\mathbf{8 0 0} \times \mathbf{8 0 0}$ pixels: $80 \times 80$ sub-apertures of $10 \times 10$ pixels each.

- Back illuminated for highest QE and best intra-pixel uniformity.

- $\quad 24 \mu \mathrm{m}$ square pixels $\quad \backslash$ Each sub-aperture is $240 \mu \mathrm{m}$ square.

- 700 fps continuous readout (1000 fps goal). \Lower frame rates/ longer integration times are also available.

- $\quad<3 \mathrm{e}^{-}$rms total readout noise.

- Nominal operating temperature $-10{ }^{\circ} \mathrm{C}$ to minimise dark current.

- $\quad$ Rolling shutter for lowest noise $\backslash$ Parallel architecture allows low noise bandwidth with high frame rate.

- On-chip ADC giving digital outputs in LVDS.

- Low cross-talk and high uniformity between pixel readout paths.

- $\quad$ Peltier-cooled hermetically-sealed package.

\subsection{Pixels and ADC architecture}

All pixels are in one continuous array; this allows use with other sub-aperture sizes. 20 dark reference columns on each side of the pixel array allow dark level to be tracked for row noise subtraction. The readout is split into upper and lower sections. Column parallel ADC blocks are used, with resolution programmable to 9 bit (fastest) or 10 bit (lowest noise). Programmable gain pre-amps are used before the ADC; each region of $40 \times 40$ pixels is independently set; four gain choices effectively add three bits and increase the dynamic range.

Each ADC block has a single row, but the channel pitch is one quarter of the pixel pitch to allow two groups of four rows of pixels to be quantised in parallel. Pixel output tracks (columns) are in sets of four (see figure below); ADC channels have great immunity to cross-talk. The sensor offers good non-synchronicity within each sub-aperture $(<2 \%)$ and low latency within each sub-aperture $(<2 \%$ of exposure time). There are 3360 parallel channels in each half sensor with LVDS outputs for image data, dark reference pixels and data synchronisation. Multiple test and diagnostic features for both factory and field use are included. See figures below for illustration of architecture.

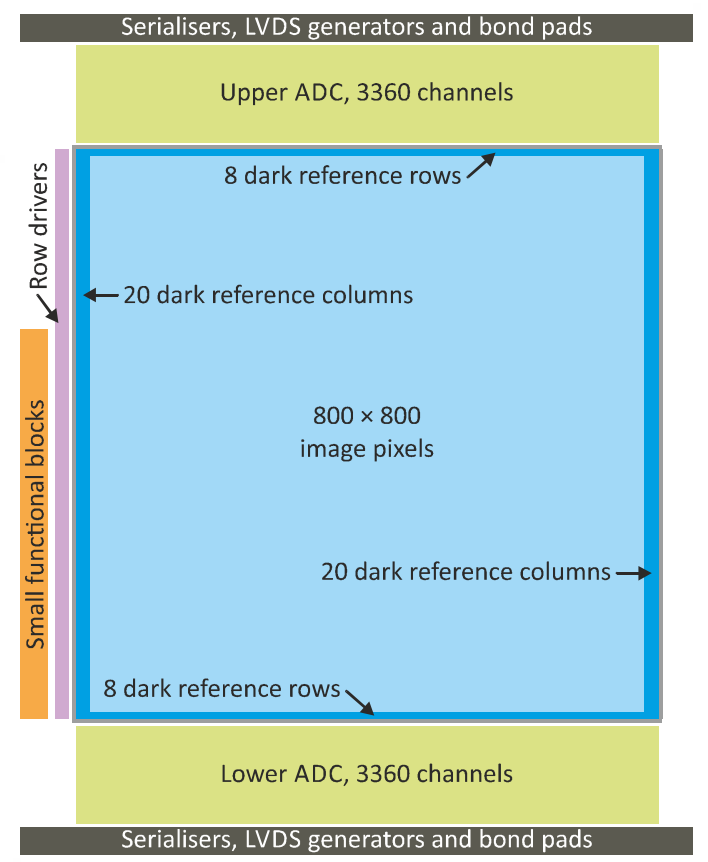

Figure 3. Device architecture

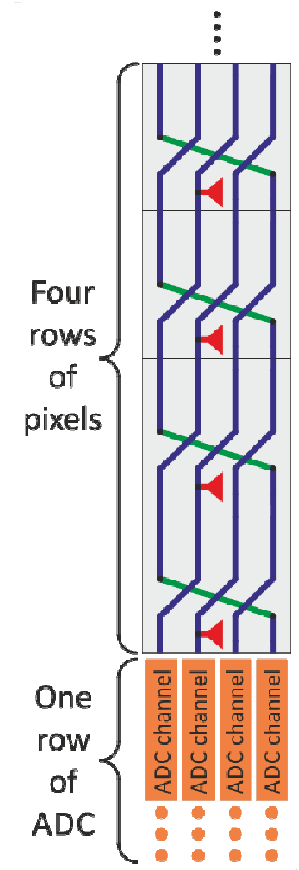

Figure 4. Tracking within pixels 


\subsection{Pixel read, data output, and timing}

During pixel read the ADC quantises all pixels of a four-row group simultaneously. Synchronicity and latency are both good: Figure 5 illustrates the read of one SA comprising 10 rows which are read in groups of four. The penultimate SA is the $96^{\text {th }}$ group, $97^{\text {th }}$ group and first half of $98^{\text {th }}$ group. The last SA is second half of $98^{\text {th }}$ group, $99^{\text {th }}$ group and then the $100^{\text {th }}$ group. The figure shows the last 24 rows together with the lower ADC block of the sensor. The upper half is a mirror image.

During data output the data is read out in raster format. Pixel values are transmitted in serial 9 (or 10) bit format.

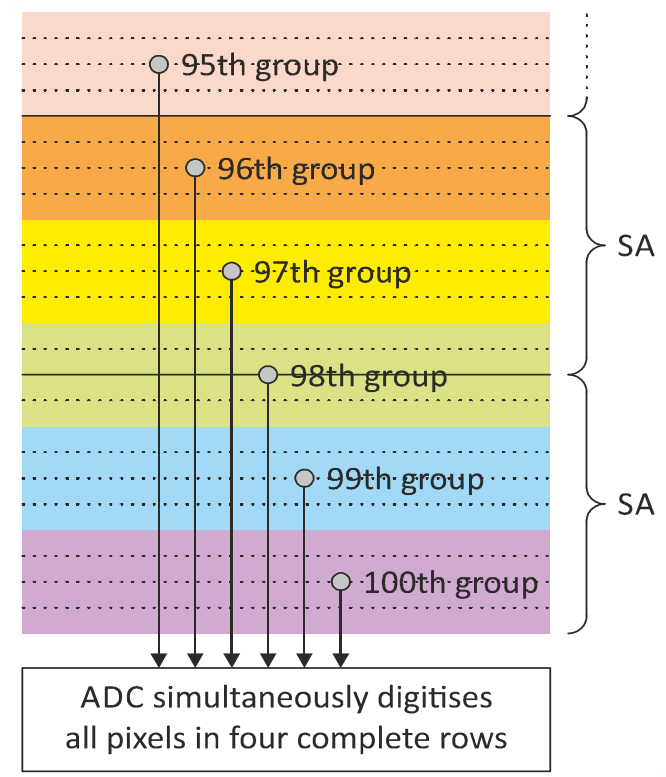

Figure 5. Sub-Aperture readout

The sensor has an on-chip pixel read timing sequencer. All pixel timing pulses, including double sampling for CDS, are generated on-chip. An on-chip ADC sequencer is provided with input pins that allow flexible start/stop ADC control. Other functions are internally generated automatically. The on-chip data output sequencer allows readout to be initiated by an input pin and then runs autonomously for each whole group of four rows- including generating the output data synchronisation signals. An optional on-chip PLL allows fast clocks to ADC and data output. The sensor is controlled via an SPI (Serial-Programmable-Interface).

\subsection{Spectral response}

With front illumination the front face features on CMOS image sensors reduce both photo-response uniformity and overall QE. The CIS124 is therefore built with back illumination and a good AR coating. This gives $90 \%$ peak quantum efficiency at visible wavelengths. These sensors have a uniform detection surface and give superior intra-pixel uniformity compared to front illuminated sensors. See Figure 6 for predicted spectral response.

Metal will be deposited on the back surface to make dark reference pixels and also to shield the read circuits from light. The figure shows an example of a CIS112 sensor with metal shield and also the non-reflective AR-coated image area. 


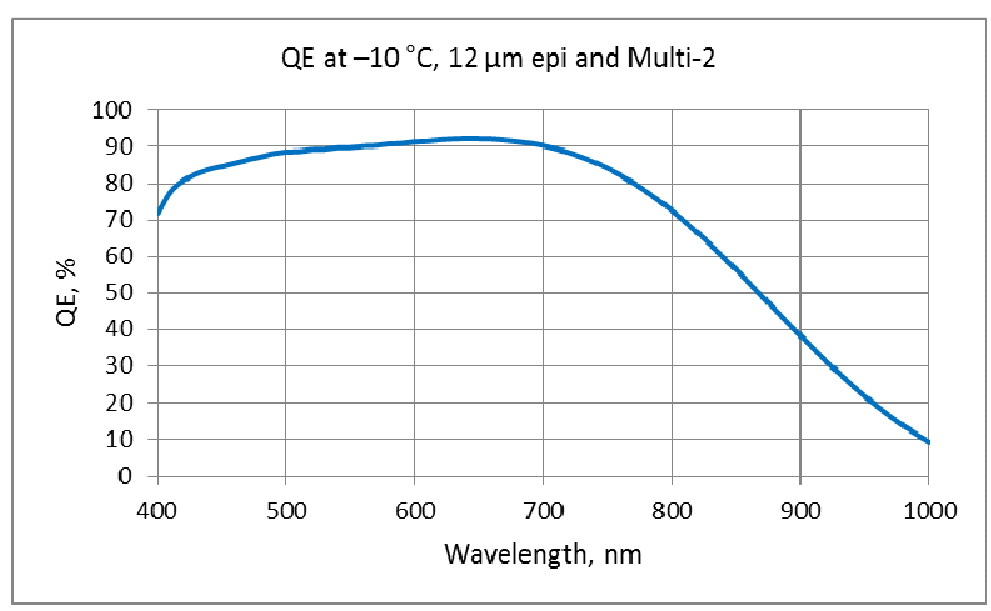

Figure 6. CIS124 spectral response

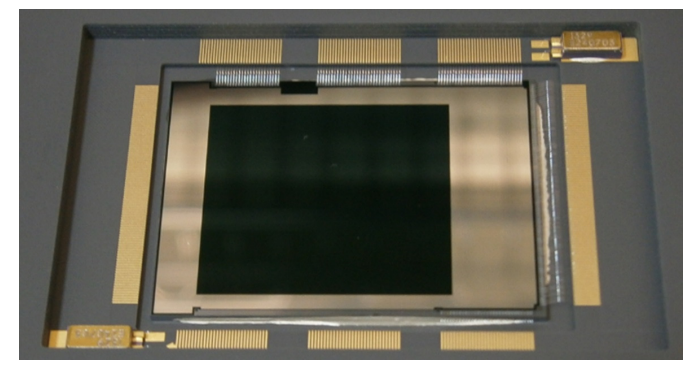

Figure 7. Illustration of metal shield [CIS112]

\subsection{Package design and construction}

The sensor design includes the following features as illustrated in the figures- A ceramic body with internal Peltier cooler (and its power feedthroughs), metal baseplate for mounting the module and for cooling the hot side of the Peltier, hermetically sealed window, low thermal conductivity inert gas filling, pinched-off pump tube after filling with inert gas, pin grid arrays each side of baseplate, temperature sensor.

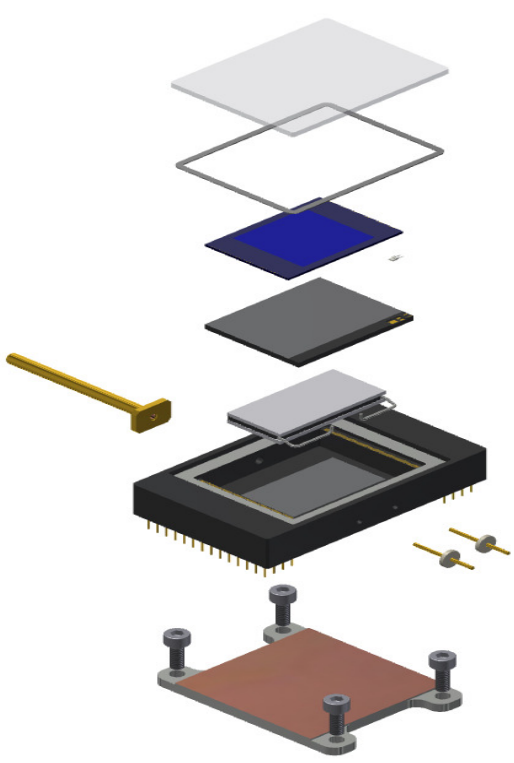

Figure 8. Exploded view of components

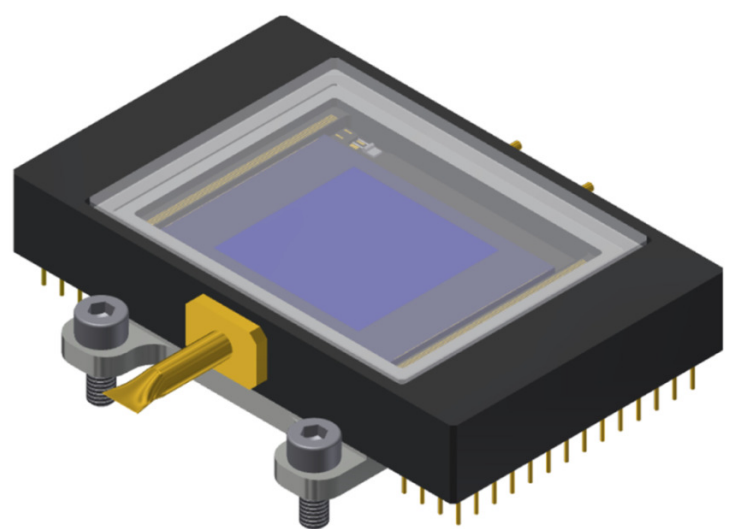

Figure 9. Illustration of finished device

\section{OTHER CMOS SENSORS FOR ASTRONOMY}

To complement the discussion above on sensors for AO we also present a few associated CMOS sensors that are relevant for potential astronomical use. 


\subsection{CIS113}

This sensor was originally designed for the TAOS-II project (Trans-Neptunian-Object detection) whereby such objects are detected by occultation of background stars ${ }^{2}$. This large-area sensor is designed for cryogenic use in mosaic focal planes and has the capability of reading out thousands of ROIs (windows) at 20 frames/second with low read-noise and high sensitivity. Forty devices have been delivered to complete the original contract. See figures and table below.
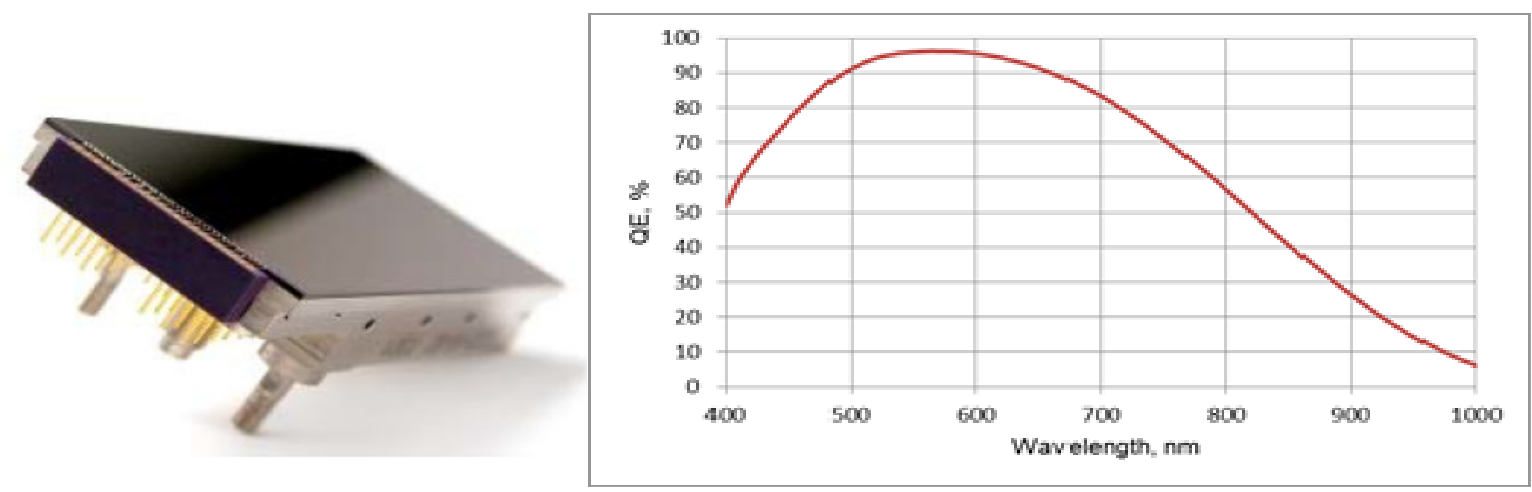

Figure 10. CIS113 sensor photo and spectral response

Table 3. CIS113 typical performance.

\begin{tabular}{|l|l|}
\hline Number of pixels & $1920(\mathrm{H}) \times 4608(\mathrm{~V})$ \\
\hline Pixel size & $16.0 \mu \mathrm{m}$ square \\
\hline Image area & $73.73 \mathrm{~m} \times 30.72 \mathrm{~mm}$ \\
\hline Output ports (analogue) & $8(\mathrm{REF}$ and SIG each) \\
\hline Package size & $82.39 \mathrm{~mm} \times 31.7 \mathrm{~mm}$ \\
\hline Package format & 76 pin ceramic PGA attached to invar base \\
\hline Focal plane height & $14.0 \mathrm{~mm}$ \\
\hline Flatness & $<30 \mu \mathrm{m}(\mathrm{peak}-$ valley) \\
\hline Conversion gain & $75 \mu \mathrm{V} / \mathrm{e}^{-}$ \\
\hline Readout noise & $3 \mathrm{e}^{-}$at $2 \mathrm{MP} / \mathrm{s}$ per channel \\
\hline Maximum pixel rate & $2 \mathrm{MP} / \mathrm{s}$ per channel \\
\hline Maximum charge & $22,000 \mathrm{e}^{-}$per pixel \\
\hline Dark signal & $70 \mathrm{e}^{-} / \mathrm{pixel} / \mathrm{s}\left(\right.$ at $\left.21^{\circ} \mathrm{C}\right)$ \\
\hline Frame rate & $2 \mathrm{fps}($ full frame mode $)$. \\
\hline
\end{tabular}

\subsection{EV76C664}

This sensor is a new standard CMOS sensor for multiple applications. It is front illuminated (with microlens) and is a fully digital sensor with multiple modes of use and low read-noise. See figure and table below.

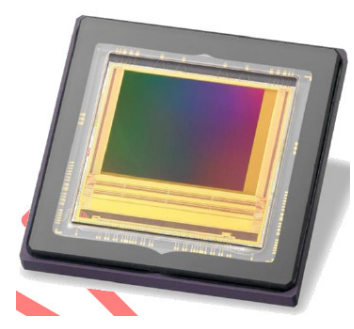

Figure 11. EV76C664.

\begin{tabular}{|l|l|}
\hline Number of pixels & $1280 \times 1024 \quad$ (1.3 Megapixel) \\
\hline Pixel size & $10.0 \mu \mathrm{m}$ square \\
\hline Shutter modes & Global and Rolling \\
\hline Output & $8,10,12,14$ bit LVDS \\
\hline Package format & Ceramic 67-pin PGA \\
\hline Readout noise & $6 \mathrm{e}^{-}$(min, depending on mode) \\
\hline Quantum Efficiency & Monochrome or sparse colour (with microlens) \\
\hline Maximum charge & $16,000 \mathrm{e}^{-} \quad$ per pixel \\
\hline
\end{tabular}

Table 4. Typical performance 


\subsection{CIS115}

This sensor is developed for space applications and is planned for use on the ESA JANUS (Juice) mission. It is backilluminated with low read-noise. Samples have been manufactured and are being qualified for space use. See figure and table below.

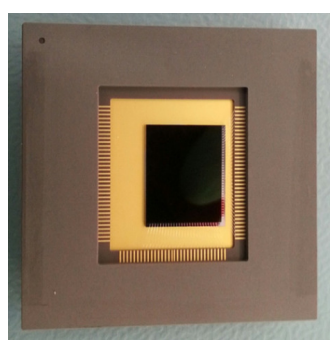

Figure 12. CIS115

\begin{tabular}{|l|l|}
\hline Number of pixels & $1504(\mathrm{H}) \times 2000(\mathrm{~V})$ \\
\hline Pixel size & $7.0 \mu \mathrm{m}$ square \\
\hline Number of output ports (reset and signal pins) & 4 pairs of analogue outputs \\
\hline Package size & $48.26 \mathrm{~mm}$ square \\
\hline Package format & 140 pin ceramic PGA \\
\hline Flatness & $<10 \mu \mathrm{m}$ (peak to valley) \\
\hline Conversion gain & $35 \mu \mathrm{V} / \mathrm{e}^{-}$ \\
\hline Readout noise & $7 \mathrm{e}^{-}($Rolling shutter) \\
\hline Maximum pixel data rate & $8 \mathrm{MP} / \mathrm{s}$ per channel \\
\hline Maximum charge per pixel & $55,000 \mathrm{e}^{-}$ \\
\hline Frame rate & Up to $10 \mathrm{~Hz}$ \\
\hline Minimum time to read one line at $6 \cdot 2 \mathrm{MP} / \mathrm{s}$ & $66.25 \mu \mathrm{s}$ \\
\hline Frame rate at full resolution & Up to $7.5 \mathrm{fps}$ \\
\hline
\end{tabular}

Table 5. CIS key parameters

\subsection{Red-sensitive CMOS}

Monolithic CMOS imagers use photodiodes biased at low voltages (1-2V) which give a small depletion depth; this means that fully depleted silicon is rather thin $(<10 \mu \mathrm{m})$ and has correspondingly small red/NIR wavelength sensitivity. This is a disadvantage compared to CCDs which use larger voltages, can achieve depletion depths in excess of $40 \mu \mathrm{m}$ and deliver high long wavelength QE. [Hybrid CMOS Imagers can deliver higher red sensitivity but are more expensive and have higher read-noise].

A modification to the design can allow reverse-biased CMOS pixels with high red wavelength spectral response as shown below. This technology is in development by Teledyne-e2v in conjunction with the CEI (Open University); acknowledgements to K Stefanov ${ }^{3}$. This principle can be applied to allow modification most existing designs. This can have value for near-IR astronomy, space imaging as well as soft-x-ray (| $10 \mathrm{keV})$ applications. Watch this space!

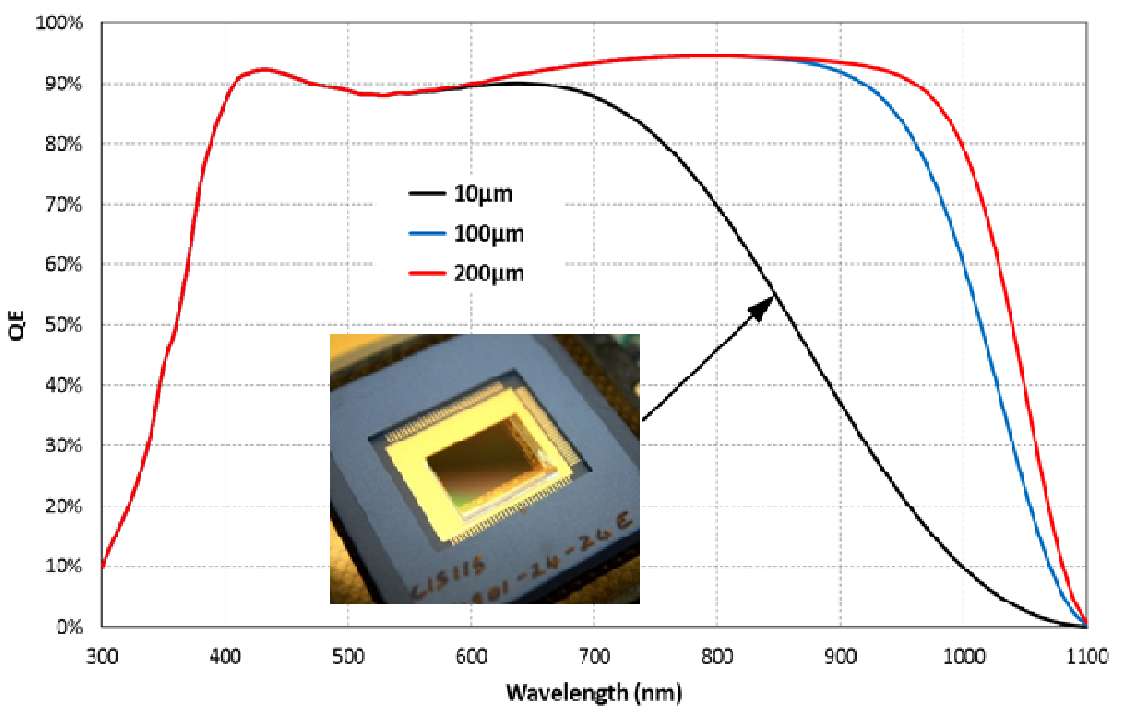

Figure 13. Spectral response of thicker silicon sensors. 


\section{SYSTEMS}

In addition to designing and manufacturing high performance sensors, Teledyne-e2 $\mathrm{v}$ also has significant capability in the design, manufacture, integration and test of sub-assemblies and systems for ground-based and space use. We illustrate below three recent examples of such projects. We therefore have the capacity to supply sub-systems such as cameras for AO WFS systems.

\subsection{J-PAS Cryocam}

The J-PAS Cryocam is a1.2 Gigapixel cryogenic camera with a $450 \mathrm{~mm}$ focal plane which is flat to $27 \mu \mathrm{m}$ at cryogenic temperature. It incorporates 14 large-area science CCDs with 224 synchronous readout channels and $<5 \mathrm{e}^{-}$noise. An integrated vacuum cryogenic system $\&$ thermal control system is included- for use on a $2.5 \mathrm{~m}$ telescope ${ }^{4}$. See figures.
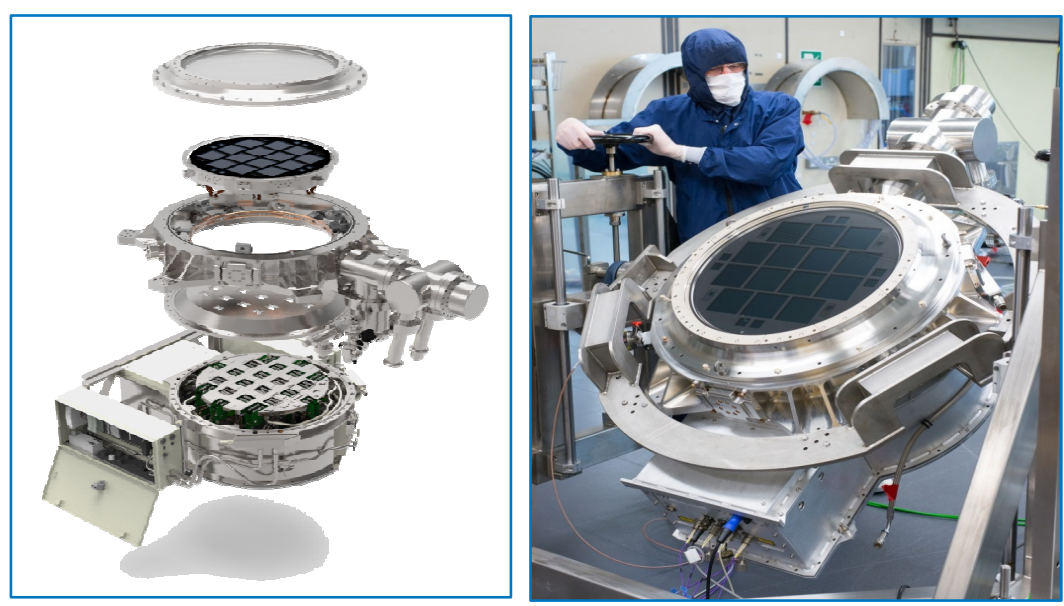

\subsection{KMTNet precision focal planes}

For the KMTNet project we have designed, assembled, and supplied three $350 \mathrm{~mm}$ focal planes ${ }^{5}$. Each precision silicon carbide plate included four large-area science CCDs and four guide sensors. The focal plane has high flatness precision and stability at cryogenic temperature. See figures below.
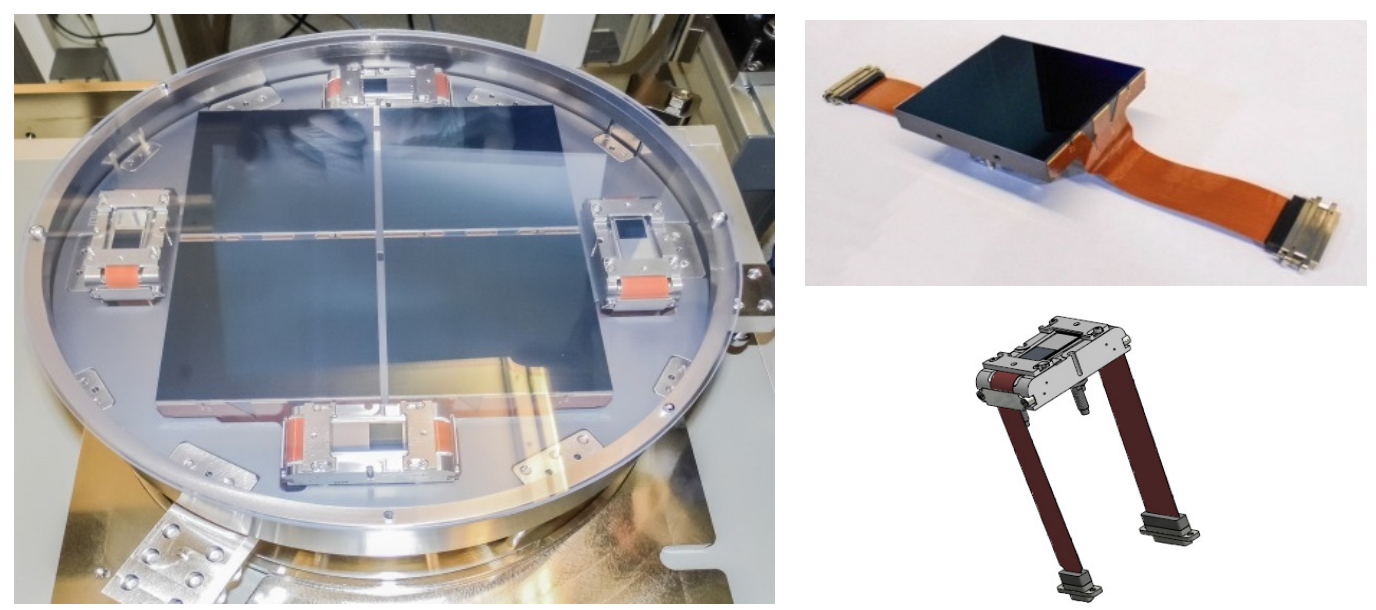

Figure 14. (a) KMTNet focal plane, (b) CCD290-99 science sensor, (c) CCD47-20 guide sensor. 


\subsection{WSO UV sensor system}

Teledyne e2v is designing custom components for the World Space Observatory UV Spectrograph- including custom EUV sensors together with space-electronics. Key components include- three custom sensor channels for 115-310 nm range, sealed vacuum cryostat enclosures for 9 year life, flight electronics (associated with RAL Space). See Figure 15.

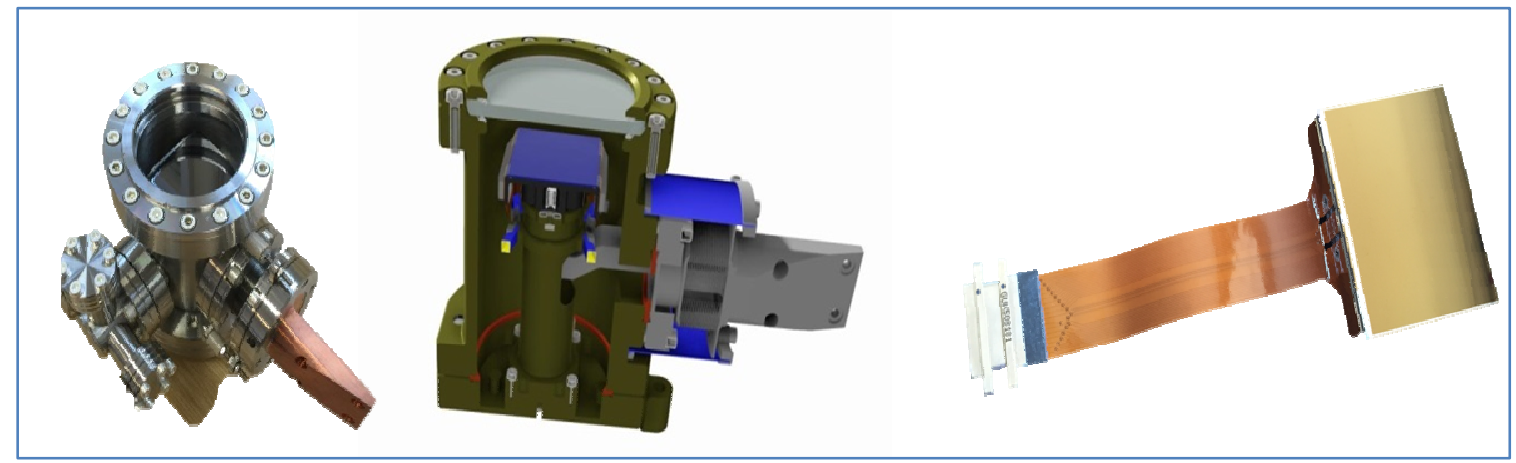

Figure 15. WSO-UV. (a) sealed sensor chamber and UV detector

\section{SUMMARY}

Teledyne $\mathrm{e} 2 \mathrm{v}$ has developed a range of specialised sensors for adaptive optics applications:

-Designed with high frame rate, high spectral response, and low noise

-Large format for ELT use; smaller formats also available; CCD and CMOS technologies

-Low latency and good uniformity for differing sub-aperture sizes

-High sensitivity for natural and laser guide star use

-Custom packages and Peltier cooling supplied

We are currently developing the next generation of large-format high frame-rate sensor for ELT use

-This "LVSM" CMOS sensor has a 2-year development timeframe.

Teledyne e $2 \mathrm{v}$ also designs and manufactures other custom CCD and CMOS sensors for astronomical use

Teledyne $\mathrm{e} 2 \mathrm{v}$ designs and supplies sub-systems and systems for ground-based and space use

\section{ACKNOWLEDGEMENTS}

Acknowledgements to multiple contributors within Teledyne-e2v and also including those from ESO (Mark Downing \& Enrico Marchetti; European Southern Observatory).

\section{REFERENCES}

[1] www.teledyne-e2v.com for datasheets and further information (CCD351, CIS113, CIS115, Onyx EV76C664, etc.).

[2] Pratlong J, et al, A 9 Megapixel large-area back-thinned CMOS sensor with high sensitivity and high frame rate for the TAOS II programme. Proc SPIE 9915, 991514 (2016).

[3] Stefanov K, et al, Fully Depleted Pinned Photodiode CMOS Image Sensor with Reverse Substrate Bias, IEEE EDL (2017).

[4] Robbins M, et al, Performance of the e2v 1.2 GPix cryogenic camera for the J-PAS 2.5m survey telescope, Proc SPIE 9908, 990811 (2016).

[5] Atwood B, et al, Design of the KMTNet large format CCD Camera, Proc SPIE 8446 (2012). 